\title{
Correction to: Characterization of Croatian Honeys by Right-Angle Fluorescence Spectroscopy and Chemometrics
}

\author{
Ivica Strelec $^{1}$ - Lidija Brodar ${ }^{2}$ Ivana Flanjak ${ }^{1}$ (D) Frane Čačić Kenjerić ${ }^{1}$. \\ Tihomir Kovač ${ }^{1}$ - Daniela Čačić Kenjerić ${ }^{1} \cdot$ Ljiljana Primorac $^{1}$
}

Published online: 17 November 2017

(C) Springer Science+Business Media, LLC, part of Springer Nature 2017

\section{Correction to: Food Anal Methods}

https://doi.org/10.1007/s12161-017-1059-z

The original version of this article unfortunately contained an incorrect version of author's names and surnames (underlined). The correct names and surnames are: "Ivica

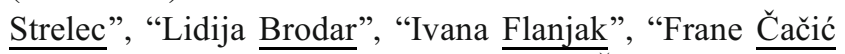
Kenjerić", "Tihomir Kovač", "Daniela Č Cačić Kenjerić", "Ljiljana Primorac".

The original article has been corrected.

The online version of the original article can be found at https://doi.org/ 10.1007/s12161-017-1059-z.

Ivana Flanjak

Ivana.Flanjak@ptfos.hr

1 Faculty of Food Technology Osijek, Josip Juraj Strossmayer University of Osijek, Franje Kuhača 20, HR-31000 Osijek, Croatia

2 Department of Biology, Josip Juraj Strossmayer University of Osijek, Ulica cara Hadrijana 8/A, HR-31000 Osijek, Croatia 